\title{
Serial relation and textural rough set
}

\author{
ŞENOL Dost
}

\begin{abstract}
The generalized rough set theory is based on the lower and upper approximation operators defined on the binary relation. The rough sets obtained from serial relations take an important place in topological applications. In this paper, we consider serial relation for texture spaces. A texturing $\mathcal{U}$ of a set $U$ is a complete and completely distributive lattice of subset of the power set $\mathcal{P}(U)$ which satisfies some certain conditions. Serial relation is defined by using textural sections and presections under a direlation on a texturing. We give some properties of serial direlation and a discussion on rough set theory from the textural point of view under serial direlation. Further, the concept of serial direlation has been characterized in terms of lower and upper textural approximation operators.
\end{abstract}

\section{INTRODUCTION}

The rough set was introduced by Pawlak in 1982 as a tool for dealing with the incomplete knowledge in information and decision systems. The main concept of the theory is the lower and upper approximation operators formed by an equivalence relation on a finite universe [14]. However, it is clear that the equivalence relation has some limitations in rough set theory applications. In order to expand the application areas of the theory, the equivalence relation has been replaced by an arbitrary binary relation. In this way, it has been the generalization of concepts of Pawlak rough set approximation operators [17-19].

A texturing $\mathcal{U}$ of a set $U$ is a complete and completely distributive lattice of subset of the power set $\mathcal{P}(U)$ which satisfies some certain conditions. It was shown that $[1-3]$ texture spaces provide a a unified setting for the study of fuzzy lattices and their topologies and bitopologies. Further direlations which are compatible the lattice structure of texture spaces defined as a suitable morphisms in categories of texture spaces. In recent years, generalizations of some concepts have been studied in texture space theory [10-13].

2020 Mathematics Subject Classification. 06D72, 26E25, 03E72.

Key words and phrases. Direlation, Texture, Fuzzy sets, Serial relation, Rough set.

Full paper. Received 19 April 2021, revised 5 May 2021, accepted 8 June 2021, available online 22 September 2021. 
In [6], textural rough set algebra was introduced to approach for generalized rough set, and it is obtained effective results for classical rough sets. Note that $\left(U, \mathcal{U}, \sigma, r^{\leftarrow}, R^{\leftarrow}\right)$ is called a textural rough set algebra where $(r, R)$ is a complemented direlation on complemented texture space $(U, \mathcal{U}, \sigma)$ and $\left(R^{\leftarrow}, r^{\leftarrow}\right)$ is inverse direlation of $(r, R)$. The pair $\left(r^{\leftarrow} A, R^{\leftarrow} A\right)$ is approximations of a set $A \in \mathcal{U}$ where $r^{\leftarrow} A$ and $R^{\leftarrow} A$ are presections of $A$. It has been studied rough sets through algebraic approach in texture theory[7-9]. The aim of this study is introduced the notion of serial direlation and analyze the corresponding textural rough sets.

In the next sections [2-4], we shall briefly the basic motivation and its study for texture spaces. For more details, we refer to [1-5].

\section{TeXture SPACES}

Let $U$ be a set. A texturing $\mathcal{U}$ of $U$ is a subset of $\mathcal{P}(U)$ which is a pointseparating, complete, completely distributive lattice containing $U$ and $\emptyset$, and for which meet coincides with intersection and finite joins with union. The pair $(U, \mathcal{U})$ is then called a texture space, or shortly texture.

For $u \in U$, the $p$-sets and, as a dually, the $q$-sets are defined by

$$
P_{u}=\bigcap\{A \in \mathcal{U} \mid u \in A\}, \quad Q_{u}=\bigvee\{A \in \mathcal{U} \mid u \notin A\} .
$$

A mapping $\sigma_{U}: \mathcal{U} \rightarrow \mathcal{U}$ is called a complementation on $(U, \mathcal{U})$ if it satisfies the conditions $\sigma_{U}\left(\sigma_{U}(A)\right)=A$ for all $A \in \mathcal{U}$ and $A \subseteq B \Longrightarrow \sigma_{U}(B) \subseteq \sigma_{U}(A)$ for all $A, B \in \mathcal{U}$. In this case $(U, \mathcal{U}, \sigma)$ is said to be complemented texture.

Example 1. (1) For any set $U,\left(U, \mathcal{P}(U), c_{U}\right)$ is the complemented discrete texture representing the usual set structure of $X$. Here the complementation $c_{U}(A)=U \backslash A, A \subseteq U$, is the usual set complement. Clearly, $P_{u}=\{u\}$ and $Q_{u}=U \backslash\{u\}$ for all $u \in U$.

(2) Let $L=(0,1], \mathcal{L}=\{(0, r] \mid r \in[0,1]\}$ and $\lambda((0, r])=(0,1-r], r \in[0,1]$. Then $(L, \mathcal{L}, \lambda)$ is complemented texture space. Here $P_{r}=Q_{r}=(0, r]$ for all $r \in L .(L, \mathcal{L})$ is said to be Hutton texture.

(3) For $\mathbb{I}=[0,1]$ define $\mathcal{J}=\{[0, t] \mid t \in[0,1]\} \cup\{[0, t) \mid t \in[0,1]\}, \iota([0, t])=$ $[0,1-t)$ and $\iota([0, t))=[0,1-t], t \in[0,1]$. Again $(\mathbb{I}, \mathcal{J}, \iota)$ is a complemented texture, which is called unit interval texture. Here $P_{t}=[0, t]$ and $Q_{t}=[0, t)$ for all $t \in I$.

(4) The product texture $(U \times V, \mathcal{U} \otimes \mathcal{V})$ of textures $(U, \mathcal{U})$ and $(V, \mathcal{V})$. Here the product texturing $\mathcal{U} \otimes \mathcal{V}$ of $U \times V$ consists of arbitrary intersections of sets of the form $(A \times V) \cup(U \times B), A \in \mathcal{U}$ and $B \in \mathcal{V}$.

For $(u, v) \in U \times V, P_{(u, v)}=P_{u} \times P_{v}$ and $Q_{(u, v)}=\left(Q_{u} \times V\right) \cup\left(U \times Q_{v}\right)$. 


\section{Direlations}

Let $(U, \mathcal{U}),(V, \mathcal{V})$ be textures. Consider the product texture $\mathcal{P}(U) \otimes \mathcal{V}$ of the textures $(U, \mathcal{P}(U))$ and $(V, \mathcal{V})$ (see Example 1(4)). We denote the $p$-sets and the $q$-sets by $\bar{P}_{(u, v)}$ and $\bar{Q}_{(u, v)}$, respectively. From the product texturing, it is obtained that

$$
\bar{P}_{(u, v)}=\{u\} \times P_{v} \quad \text { and } \quad \bar{Q}_{(u, v)}=(U \backslash\{u\} \times V) \cup\left(U \times Q_{v}\right)
$$

where $u \in U$ and $v \in V$. It is easy to see that $\bar{P}_{(u, v)} \nsubseteq \bar{Q}_{\left(u^{\prime}, v^{\prime}\right)}$ implies $u=u^{\prime}$ and $P_{v} \nsubseteq Q_{v^{\prime}}$

Then:

(1) $r \in \mathcal{P}(U) \otimes \mathcal{V}$ is called a relation from $(U, \mathcal{U})$ to $(V, \mathcal{V})$ if it satisfies: R1 $r \nsubseteq \bar{Q}_{(u, v)}, P_{u^{\prime}} \nsubseteq Q_{u}$ implies $r \nsubseteq \bar{Q}_{\left(u^{\prime}, v\right)}$.

R2 $r \nsubseteq \bar{Q}_{(u, v)}$ implies $\exists u^{\prime} \in U$ such that $P_{u} \nsubseteq Q_{u^{\prime}}$ and $r \nsubseteq \bar{Q}_{\left(u^{\prime}, v\right)}$.

(2) $R \in \mathcal{P}(U) \otimes \mathcal{V}$ is called a corelation from $(U, \mathcal{U})$ to $(V, \mathcal{V})$ if it satisfies CR1 $\bar{P}_{(u, v)} \nsubseteq R, P_{u} \nsubseteq Q_{u^{\prime}}$ implies $\bar{P}_{\left(u^{\prime}, v\right)} \nsubseteq R$.

CR2 $\bar{P}_{(u, v)} \nsubseteq R$ implies $\exists u^{\prime} \in U$ such that $P_{u^{\prime}} \nsubseteq Q_{u}$ and $\bar{P}_{\left(u^{\prime}, v\right)} \nsubseteq R$.

(3) A pair $(r, R)$, where $r$ is a relation and $R$ a corelation from $(U, \mathcal{U})$ to $(V, \mathcal{V})$ is called a direlation from $(U, \mathcal{U})$ to $(V, \mathcal{V})$.

Examples 2. (1) The identity direlation $(i, I)$ on $(U, \mathcal{U})$ is defined by

$$
i=\bigvee\left\{\bar{P}_{(u, u)} \mid u \in U\right\} \text { and } I=\bigcap\left\{\bar{Q}_{(u, u)} \mid U \nsubseteq Q_{u}\right\} \text {. }
$$

(2) Let $(r, R)$ be a direlation from the discrete texture $(U, \mathcal{P}(U))$ to the discrete texture $(V, \mathcal{P}(V))$. Since $\mathcal{P}(U \times V)=\mathcal{P}(U) \otimes \mathcal{P}(V), r$ and $R$ are point relation from $U$ to $V$.

Inverses of a direlation: The inverses of $r$ and $R$ are defined by

$$
r^{\leftarrow}=\bigcap\left\{\bar{Q}_{(v, u)} \mid r \nsubseteq \bar{Q}_{(u, v)}\right\} \text { and } R^{\leftarrow}=\bigvee\left\{\bar{P}_{(v, u)} \mid \bar{P}_{(u, v)} \nsubseteq R\right\}
$$

respectively, where $r^{\leftarrow}$ is a corelation and $R^{\leftarrow}$ is a relation.

The direlation $(r, R)^{\leftarrow}=\left(R^{\leftarrow}, r^{\leftarrow}\right)$ from $(V, \mathcal{V})$ to $(U, \mathcal{U})$ is called the inverse of the direlation $(r, R)$.

The complement of a direlation: Let $(r, R)$ be a direlation between the complemented textures $\left(U, \mathcal{U}, \sigma_{U}\right)$ and $\left(V, \mathcal{V}, \sigma_{V}\right)$.

(1) The complement $r^{\prime}$ of the relation $r$ is the co-relation

$$
\begin{gathered}
r^{\prime}=\bigcap\left\{\bar{Q}_{(u, v)} \mid \exists w, z,\right. \\
\left.r \nsubseteq \bar{Q}_{(w, z)}, \sigma_{U}\left(Q_{u}\right) \nsubseteq Q_{w} \text { and } P_{z} \nsubseteq \sigma_{V}\left(P_{v}\right)\right\} .
\end{gathered}
$$


(2) The complement $R^{\prime}$ of the co-relation $R$ is the relation

$$
\begin{gathered}
R^{\prime}=\bigvee\left\{\bar{P}_{(u, v)} \mid \exists w, z, \bar{P}_{(w, z)} \nsubseteq R,\right. \\
\left.P_{w} \nsubseteq \sigma_{U}\left(P_{u}\right) \text { and } \sigma_{V}\left(Q_{v}\right) \nsubseteq Q_{z}\right\} .
\end{gathered}
$$

(3) The complement $(r, R)^{\prime}$ of the direlation $(r, R)$ is the direlation $(r, R)^{\prime}=\left(R^{\prime}, r^{\prime}\right)$

A direlation $(r, R)$ on $(U, \mathcal{U})$ is said to be complemented if $(r, R)^{\prime}=(r, R)$.

Order between Direlations: Let $\left(r_{1}, R_{1}\right)$ and $\left(r_{2}, R_{2}\right)$ be direlations from $(U, \mathcal{U})$ to $(V, \mathcal{V})$. The inclusion $\sqsubseteq$ between direlations is defined by

$$
\left(r_{1}, R_{1}\right) \sqsubseteq\left(r_{2}, R_{2}\right) \quad \text { if } \quad r_{1} \subseteq r_{2} \text { and } R_{2} \subseteq R_{1} .
$$

A direlation $(r, R)$ on $(U, \mathcal{U})$ is called:

(i) reflexive if $(i, I) \sqsubseteq(r, R)$,

(ii) symmetric if $(r, R)^{\leftarrow}=(r, R)$,

(iii) transitive if $(r, R) \circ(r, R) \sqsubseteq(r, R)$.

\section{Sections And PRESECtions}

Let us recall that[4] some properties of sections and presections are given in this subsection.

Let $(r, R)$ be a direlation on $(U, \mathcal{U})$ and $A, B \in \mathcal{U}$. The $A$-sections under $(r, R)$ are given as:

$$
\begin{aligned}
& r \rightarrow A=\bigcap\left\{Q_{v} \mid \forall u, r \nsubseteq \bar{Q}_{(u, v)} \Longrightarrow A \subseteq Q_{u}\right\}, \\
& R^{\rightarrow} A=\bigvee\left\{P_{v} \mid \forall u, \bar{P}_{(u, v)} \nsubseteq R \Longrightarrow P_{u} \subseteq A\right\} .
\end{aligned}
$$

Likewise, the $B$-presections under $(r, R)$ are given as

$$
\begin{aligned}
& r^{\leftarrow} B=\bigvee\left\{P_{u} \mid \forall v, r \nsubseteq \bar{Q}_{(u, v)} \Longrightarrow P_{v} \subseteq B\right\}, \\
& R^{\leftarrow} B=\bigcap\left\{Q_{u} \mid \forall v, \bar{P}_{(u, v)} \nsubseteq R \Longrightarrow B \subseteq Q_{v}\right\} .
\end{aligned}
$$

Note that section and presection are related as in the next result:

Lemma 3. Let $(r, R)$ be a direlation $(U, \mathcal{U})$ and $A \in \mathcal{U}$. Then:

$$
\begin{array}{cll}
A \subseteq r^{\leftarrow}\left(r^{\rightarrow} A\right) & \text { and } & r^{\rightarrow}\left(r^{\leftarrow} A\right) \subseteq A, \\
R^{\leftarrow}\left(R^{\rightarrow} A\right) \subseteq A & \text { and } & A \subseteq R^{\rightarrow}\left(R^{\leftarrow} A\right), \\
\left(r^{\leftarrow}\right)^{\leftarrow} A=r^{\rightarrow} A & \text { and } & \left(R^{\leftarrow}\right)^{\leftarrow} A=R^{\rightarrow} A .
\end{array}
$$

Some basic properties of sections and presections such as inclusion and meet and join are given below. 
Lemma 4. Let $A, B \in \mathcal{U}$ and $\left\{A_{j} \mid j \in J\right\} \subseteq \mathcal{U}$. Then sections and presections satisfy the following properties.
(i) $r \rightarrow \emptyset=\emptyset$ and $R^{\rightarrow} U=U$,
$r^{\leftarrow} U=U$ and $R^{\leftarrow} \emptyset=\emptyset$
(ii) $A \subseteq B \Longrightarrow r^{\rightarrow} A \subseteq r^{\rightarrow} B$, $A \subseteq B \Longrightarrow r^{\leftarrow} A \subseteq r^{\leftarrow} B$
(iii) $A \subseteq B \Longrightarrow R^{\rightarrow} A \subseteq R^{\rightarrow} B$, $A \subseteq B \Longrightarrow R^{\leftarrow} A \subseteq R^{\leftarrow} B$
(iv) $r \rightarrow\left(\bigvee_{j \in J} A_{j}\right)=\bigvee_{j \in J} r^{\rightarrow} A_{j}$, $\bigvee_{j \in J} r^{\leftarrow} A_{j} \subseteq r^{\leftarrow}\left(\bigvee_{j \in J} A_{j}\right)$
(v) $r \rightarrow\left(\bigcap_{j \in J} A_{j}\right) \subseteq \bigcap_{j \in J} r^{\rightarrow} A_{j}$,
$r^{\leftarrow}\left(\bigcap_{j \in J} A_{j}\right)=\bigcap_{j \in J} r^{\rightarrow} A_{j}$
(vi) $\bigvee_{j \in J} R^{\rightarrow} A_{j} \subseteq R^{\rightarrow}\left(\bigvee_{j \in J} A_{j}\right)$,
$R^{\leftarrow}\left(\bigvee_{j \in J} A_{j}\right)=\bigvee_{j \in J} R^{\leftarrow} A_{j}$
(vii) $R^{\rightarrow}\left(\bigcap_{j \in J} A_{j}\right)=\bigcap_{j \in J} R^{\rightarrow}\left(A_{j}\right)$,
$R^{\leftarrow}\left(\bigcap_{j \in J} A_{j}\right) \subseteq \bigcap_{j \in J} R^{\leftarrow} A_{j}$

Lemma 5. Let $(r, R)$ be a direlation on a complemented texture $(U, \mathcal{U}, \sigma)$. For $A \in \mathcal{U}$, hold

(i) $\left(r^{\prime}\right) \rightarrow A=\sigma\left(r^{\rightarrow} \sigma(A)\right)$ and $\left(R^{\prime}\right) \rightarrow A=\sigma\left(R^{\rightarrow} \sigma(A)\right)$,

(ii) $\left(r^{\prime}\right)^{\leftarrow} A=\sigma\left(r^{\leftarrow} \sigma(A)\right)$ and $\left(R^{\prime}\right)^{\leftarrow} A=\sigma\left(R^{\leftarrow} \sigma(A)\right)$.

From [11, Proposition 4.4.], we have:

Proposition 6. Let $(r, R)$ be a direlation on a texture $(U, \mathcal{U})$. Then:

(i) $(r, R)$ is reflexive if and only if $r^{\leftarrow} A \subseteq A \subseteq R^{\leftarrow} A$, for all $A \in \mathcal{U}$.

(ii) $(r, R)$ is transitive if and only if $r^{\leftarrow} A \subseteq r^{\leftarrow} r^{\leftarrow} A$ and $R^{\leftarrow} R^{\leftarrow} A \subseteq$ $R^{\leftarrow} A$ for all $A \in \mathcal{U}$.

(iii) If $(r, R)$ is symmetric then we have $R^{\leftarrow} r^{\leftarrow} A \subseteq A \subseteq r^{\leftarrow} R^{\leftarrow} A$ for all $A \in \mathcal{U}$.

\section{Serial Direlation}

As is known, in the rough set theory, the characterizations of reflexive and transitive binary relations are given by the lower and upper approximation operators [18]. This leads to the following serialness concepts for a direlation on texture spaces.

Definition 7. Let $(r, R)$ be a direlation on $(U, \mathcal{U})$. Then

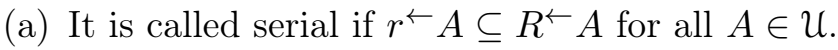

(b) It is called inverse serial if $R \rightarrow A \subseteq r \rightarrow A$ for all $A \in \mathcal{U}$.

Proposition 8. Let $(r, R)$ be a direlation on $(U, \mathcal{U})$.

(i) If $(r, R)$ is reflexive then it is serial.

(ii) If $(r, R)$ is serial and symmetric and transitive then it is reflexive. 
Proof. (i) Let $(r, R)$ be a reflexive direlation on $(U, \mathcal{U})$. Since $r^{\leftarrow}(A) \subseteq A \subseteq$ $R^{\leftarrow}(A)$ for all $A \in \mathcal{U},(r, R)$ is a serial direlation.

(ii) Let $(r, R)$ be a serial and symmetric and transitive direlation on $(U, \mathcal{U})$. For $A \in \mathcal{U}$,

$$
\begin{array}{rlrl}
r^{\leftarrow} A & \subseteq r^{\leftarrow} r^{\leftarrow} A, & R^{\leftarrow} A & \supseteq R^{\leftarrow} R^{\leftarrow} A \quad \text { (transitivity) } \\
\subseteq R^{\leftarrow} r^{\leftarrow} A & \supseteq r^{\leftarrow} R^{\leftarrow} A(\text { serialness }) \\
\subseteq A, & \supseteq A & (\text { symmetricalness })
\end{array}
$$

Hence, we have $r^{\leftarrow} A \subseteq R^{\leftarrow} A$, for all $A \in \mathcal{U}$.

Lemma 9. Let $(r, R)$ be a direlation on $(U, \mathcal{U})$. We have:

(i) $(r, R)$ is serial if and only if $r^{\leftarrow} \emptyset=\emptyset \Longleftrightarrow R^{\leftarrow} U=U$.

(ii) $(r, R)$ is inverse serial if and only if $R \rightarrow \emptyset=\emptyset \Longleftrightarrow r \rightarrow U=U$.

Proof. Suppose that $(r, R)$ is a direlation on $(U, \mathcal{U})$.

(i) From Lemma $4, R^{\leftarrow} \emptyset=\emptyset$ and $r^{\leftarrow} U=U$. Then

$$
\begin{aligned}
(r, R) \text { is serial } & \Longleftrightarrow r^{\leftarrow} A \subseteq R^{\leftarrow} A, \quad \forall A \in \mathcal{U} \\
& \Longleftrightarrow r^{\leftarrow} \emptyset \subseteq R^{\leftarrow} \emptyset, \quad \emptyset \in \mathcal{U} \\
& \Longleftrightarrow r^{\leftarrow} \emptyset \subseteq \emptyset \\
& \Longleftrightarrow r^{\leftarrow} \emptyset=\emptyset \\
(r, R) \text { is serial } & \Longleftrightarrow r^{\leftarrow} A \subseteq R^{\leftarrow} A, \quad \forall A \in \mathcal{U} \\
& \Longleftrightarrow r^{\leftarrow} U \subseteq R^{\leftarrow} U, \quad U \in \mathcal{U} \\
& \Longleftrightarrow U \subseteq R^{\leftarrow} U \\
& \Longleftrightarrow R^{\leftarrow} U=U
\end{aligned}
$$

(ii) From Lemma $4, r \rightarrow \emptyset=\emptyset$ and $R^{\rightarrow} U=U$. Then

$$
\begin{aligned}
(r, R) \text { is inverse serial } & \Longleftrightarrow R^{\rightarrow} A \subseteq r^{\rightarrow} A, \quad \forall A \in \mathcal{U} \\
& \Longleftrightarrow R^{\rightarrow} \emptyset \subseteq r^{\rightarrow} \emptyset, \quad \emptyset \in \mathcal{U} \\
& \Longleftrightarrow R^{\rightarrow} \emptyset \subseteq \emptyset \\
& \Longleftrightarrow R^{\rightarrow} \emptyset=\emptyset \\
(r, R) \text { is inverse serial } & \Longleftrightarrow R^{\rightarrow} A \subseteq r^{\rightarrow} A, \quad \forall A \in \mathcal{U} \\
& \Longleftrightarrow R^{\rightarrow} U \subseteq r^{\rightarrow} U, \quad U \in \mathcal{U} \\
& \Longleftrightarrow U \subseteq r^{\leftarrow} U \\
& \Longleftrightarrow r^{\rightarrow} U=U . \quad \square
\end{aligned}
$$

Proposition 10. Let $(r, R)$ be a symmetric direlation on $(U, \mathcal{U})$. Then $(r, R)$ is a serial direlation if and only if it is a inverse serial direlation. 
Proof. We suppose that $(r, R)$ is a symmetric direlation on $(U, \mathcal{U})$. Since $(r, R)=(r, R)^{\leftarrow}$, we have $r^{\leftarrow} A=R^{\leftarrow} A$ and $R^{\leftarrow} A=r^{\leftarrow} A$ for all $A \in \mathcal{U}$. Then, for $A \in \mathcal{U}$ :

$$
\begin{aligned}
(r, R) \text { is serial } & \Longleftrightarrow r^{\leftarrow} A \subseteq R^{\leftarrow A} \\
& \Longleftrightarrow R^{\rightarrow} A \subseteq r^{\rightarrow} A \\
& \Longleftrightarrow(r, R) \text { is inverse serial. }
\end{aligned}
$$

Corollary 11. Let $(r, R)^{\leftarrow}$ be inverse direlation of $(r, R)$. Then $(r, R)$ is (inverse-) serial if and only if $(r, R)^{\leftarrow}$ is (inverse-) serial.

Proof. Suppose that $(r, R)^{\leftarrow}$ is inverse direlation of $(r, R)$. Then $(r, R)^{\leftarrow}$ $=\left(R^{\leftarrow}, r^{\leftarrow}\right)$, and $\left(r^{\leftarrow}\right)^{\leftarrow} A=r^{\rightarrow} A, \quad\left(R^{\leftarrow}\right)^{\leftarrow} A=R^{\rightarrow} A$, for all $A \in \mathcal{U}$, from Lemma 3. Further, $(r, R)^{\leftarrow}=\left(R^{\leftarrow}, r^{\leftarrow}\right)$ is serial if and only if $\left(R^{\leftarrow}\right)^{\leftarrow} A=$ $R^{\rightarrow} A \subseteq\left(r^{\leftarrow}\right)^{\leftarrow} A=r^{\rightarrow} A$. Likewise, $(r, R)^{\leftarrow}=\left(R^{\leftarrow}, r^{\leftarrow}\right)$ is inverse serial if and only if $\left(r^{\leftarrow}\right)^{\leftarrow} A=r^{\rightarrow} A \subseteq\left(R^{\leftarrow}\right)^{\leftarrow} A=R^{\rightarrow} A$. Consequently, the proof is completed.

Proposition 12. Let $(r, R)^{\prime}$ be complement direlation of $(r, R)$ on a complemented texture $(U, \mathcal{U}, \sigma)$. Then

(i) $(r, R)$ is serial if and only if $(r, R)^{\prime}$ is serial.

(ii) $(r, R)$ is inverse serial if and only if $(r, R)^{\prime}$ is inverse serial.

Proof. Suppose that $(r, R)^{\prime}=\left(R^{\prime}, r^{\prime}\right)$ is complement direlation of $(r, R)$. From Lemma 5, $\left(R^{\prime}\right)^{\leftarrow} A=\sigma\left(R^{\leftarrow} \sigma(A)\right)$ and $\left(r^{\prime}\right)^{\leftarrow} A=\sigma\left(r^{\leftarrow} \sigma(A)\right)$ for all $A \in \mathcal{U}$. Then

(i) Let $A \in \mathcal{U}$. Then $\sigma(A) \in \mathcal{U}$.

$$
\begin{aligned}
(r, R) \text { is serial } & \Longleftrightarrow r^{\leftarrow} \sigma(A) \subseteq R^{\leftarrow} \sigma(A) \\
& \Longleftrightarrow \sigma\left(R^{\leftarrow} \sigma(A)\right) \subseteq \sigma\left(r^{\leftarrow} \sigma(A)\right) \\
& \Longleftrightarrow\left(R^{\prime}\right)^{\leftarrow}(A) \subseteq\left(r^{\prime}\right)^{\leftarrow}(A) \\
& \Longleftrightarrow\left(R^{\prime}, r^{\prime}\right) \text { is serial. }
\end{aligned}
$$

(ii) Let $A \in \mathcal{U}$. Then $\sigma(A) \in \mathcal{U}$.

$$
\begin{aligned}
(r, R) \text { is inverse serial } & \Longleftrightarrow R^{\rightarrow} \sigma(A) \subseteq r^{\rightarrow} \sigma(A) \\
& \Longleftrightarrow \sigma\left(r^{\rightarrow} \sigma(A)\right) \subseteq \sigma\left(R^{\leftarrow} \sigma(A)\right) \\
& \Longleftrightarrow\left(r^{\prime}\right) \rightarrow(A) \subseteq\left(R^{\prime}\right) \rightarrow(A) \\
& \Longleftrightarrow\left(R^{\prime}, r^{\prime}\right) \text { is inverse serial. }
\end{aligned}
$$

Let $(U, \mathcal{U}, \sigma)$ be a complemented texture and $L, H: \mathcal{U} \rightarrow \mathcal{U}$ be two unary operators. Recall that [6] $L$ and $H$ are called dual operators on $(U, \mathcal{U})$ if $L(A)=H(\sigma(A))$ and $H(A)=L(\sigma(A))$ for all $A \in \mathcal{U}$. Now, we consider the following property: 


$$
\begin{array}{ll}
\left(\mathbf{L}_{\mathbf{1}}\right) L(U)=U, & \left(\mathbf{H}_{\mathbf{1}}\right) H(\emptyset)=\emptyset, \\
\left(\mathbf{L}_{\mathbf{2}}\right) L\left(\bigcap_{j \in J} A_{j}\right)=\bigcap_{j \in J} L\left(A_{j}\right), & \left(\mathbf{H}_{\mathbf{2}}\right) H\left(\bigvee_{j \in J} A_{j}\right)=\bigvee_{j \in J} H\left(A_{j}\right) .
\end{array}
$$

Then we have $\left(\mathbf{L}_{\mathbf{1}}\right) \Longleftrightarrow\left(\mathbf{H}_{\mathbf{1}}\right)$ and $\left(\mathbf{L}_{\mathbf{2}}\right) \Longleftrightarrow\left(\mathbf{H}_{\mathbf{2}}\right)$, for the dual operators $L, H: \mathcal{U} \rightarrow \mathcal{U}$ on the texture $(U, \mathcal{U}, \sigma)$.

Recall that [6] if the dual operators $L$ and $H$ satisfy conditions $\left(\mathbf{L}_{\mathbf{1}}\right)$ and $\left(\mathbf{L}_{\mathbf{2}}\right)$ (or equivalently, $\left(\mathbf{H}_{\mathbf{1}}\right)$ and $\left(\mathbf{H}_{\mathbf{2}}\right)$ ), then the system $(U, \mathcal{U}, \sigma, L, H)$ is called a textural rough set algebra, and the operators $L$ and $H$ are called approximation operators on $(U, \mathcal{U})$. In this case, the pair $(L(A), H(A))$ is called textural rouhg set for $A \in \mathcal{U}$ where $L(A)$ is lower rough set and $H(A)$ is upper rough set.

Note that if $(r, R)$ is a complemented direlation on $(U, \mathcal{U}, \sigma)$, then the system $\left(U, \mathcal{U}, \sigma, r^{\leftarrow}, R^{\leftarrow}\right)$ is a textural rough set algebra where $r^{\leftarrow}, R^{\leftarrow}: \mathcal{U} \rightarrow \mathcal{U}$ are approximation operators.

Proposition 13. Let $L, H: \mathcal{U} \rightarrow \mathcal{U}$ be dual operators on the complemented texture $(U, \mathcal{U}, \sigma)$. Then there exists a unique complemented serial direlation $(r, R)$ on $(U, \mathcal{U}, \sigma)$ such that $L(A)=r^{\leftarrow} A$ and $\left(H(A)=R^{\leftarrow} A\right.$ for all $A \in \mathcal{U}$ if and only if $L$ and $H$ satisfy $\left(\mathbf{L}_{\mathbf{1}}\right)$ and $\left(\mathbf{L}_{\mathbf{2}}\right)$ (or equivalently, $\left(\mathbf{H}_{\mathbf{1}}\right)$ and $\left(\mathbf{H}_{\mathbf{2}}\right)$ ) and the following property:

$$
\text { (D) } L(A) \subseteq H(A), \quad \forall A \in \mathcal{U} .
$$

Proof. Let $L, H: \mathcal{U} \rightarrow \mathcal{U}$ be dual operators on the complemented texture $(U, \mathcal{U}, \sigma)$.

$(\Longrightarrow)$ First, we suppose that $(r, R)$ is a complemented serial direlation on a complemented texture $(U, \mathcal{U}, \sigma)$ such that $L(A)=r^{\leftarrow} A$ and $H(A)=R^{\leftarrow} A$ for all $A \in \mathcal{U}$. From Lemma 4 , we have $L(U)=r^{\leftarrow} U=U$ and $L\left(\bigcap_{j \in J} A_{j}\right)=$ $r^{\leftarrow}\left(\bigcap_{j \in J} A_{j}\right)=\left(\bigcap_{j \in J} r^{\leftarrow} A_{j}=\bigcap_{j \in J} L\left(A_{j}\right)\right.$. Hence, the operator $L$ satisfies $\left(\mathbf{L}_{\mathbf{1}}\right)$ and $\left(\mathbf{L}_{\mathbf{2}}\right)$. Since $\sigma\left(r^{\leftarrow} A\right)=R^{\leftarrow}(\sigma(A)), r^{\leftarrow}, R^{\leftarrow}: \mathcal{U} \rightarrow \mathcal{U}$ are dual operators, and so the operator $H$ satisfies $\left(\mathbf{H}_{\mathbf{1}}\right)$ and $\left(\mathbf{H}_{\mathbf{2}}\right)$. Finally, since $(r, R)$ is serial direlation we have $L(A)=r^{\leftarrow} A \subseteq R^{\leftarrow} A=H(A)$ for all $A \in \mathcal{U}$. Consequently, the property (D) is also satisfied.

Otherwise, we suppose that the dual operators $L$ and $H$ satisfy $\left(\mathbf{L}_{\mathbf{1}}\right)$ and $\left(\mathbf{L}_{\mathbf{2}}\right)$ and $(\mathbf{D})$. It was proved that $[6]$ the equalities

$$
\begin{aligned}
r & =\bigvee\left\{\bar{P}_{(u, v)} \mid u, v \in U, P_{u} \nsubseteq L\left(Q_{v}\right)\right\} \\
R & =\bigcap\left\{\bar{Q}_{(u, v)} \mid u, v \in U, H\left(Q_{v}\right) \nsubseteq Q_{u}\right\}
\end{aligned}
$$


define a unique complemented direlation on $(U, \mathcal{U})$ such that $L(A)=r^{\leftarrow} A$ and $\left(H(A)=R^{\leftarrow} A\right.$ for all $A \in \mathcal{U}$. Further, $(r, R)$ is serial direlation, since the operators $L$ and $H$ satisfy the property $(\mathbf{D})$.

Note 14. From the Lemma 9, the property (D) can be replaced

$$
\begin{array}{ll}
(\text { L3) } L(\emptyset) & =\emptyset, \\
\text { (H3) } H(U) & =U .
\end{array}
$$

\section{Serial RELATion AND DiRElation}

In this section, we observe the relationship between a serial binary relation and direlation.

Let $r$ be a binary relation on a universe $U$. Then it is called serial if for each $u \in U$, there exists $v \in U$ such that $(u, v) \in U ; r$ is inverse serial if for each $u \in U$, there exists $v \in U$ such that $(v, u) \in U[18]$.

Now let $r$ be a binary relation on a universe $U$. Then the pair $(U, r)$ is called a generalized approximation space. The operators $\underline{a p p}_{r}, \overline{a p p}_{r}: \mathcal{P}(U) \rightarrow \mathcal{P}(U)$ defined by

$$
\begin{aligned}
& \underline{a p p}_{r} A=\{u \in U \mid r(u) \subseteq A\}, \\
& \overline{\overline{a p p}}_{r} A=\{u \in U \mid r(u) \cap A \neq \emptyset\}, \quad A \subseteq U
\end{aligned}
$$

are called lower approximation operator and upper approximation operator, respectively where $r(u)=\{v \in U \mid(u, v) \in r\}$. Then the pair $\left(\underline{a p p}_{r} A, \overline{a p p}_{r} A\right)$ is called the rough set of $A$ [18]. If the relation $r$ is serial, then the rough set is serial rough set. Further, $r$ is serial if and only if $\underline{a p p}_{r} A \subseteq \overline{a p p}_{r} A$ for all $A \subseteq U$.

Recall that [6] if $(r, R)$ is a complemented direlation on $(U, \mathcal{U})$ then $\left(U, \mathcal{U}, r^{\leftarrow}, R^{\leftarrow}\right)$ is called complemented textural approximation space. Then $r^{\leftarrow}$ and $R^{\leftarrow}$ are approximation operators on $(U, \mathcal{U}, \sigma)$, and so the pair $\left(r^{\leftarrow} A, R^{\leftarrow} A\right)$ is textural rough set of $A \in \mathcal{U}$.

Now we observe that [4] if $r$ be a point relation on a set $U$, then the pair $(r, R)$ is a direlation on the discrete texture $(U, \mathcal{P}(U))$ where $R=(U \times U) \backslash r$. Note that $(r, R)$ is complemented and the inverse direlation $(r, R)^{\leftarrow}=\left(R^{\leftarrow}, r^{\leftarrow}\right)$ can be stated by inverse point relation such that $R^{\leftarrow}=r^{-1}$ and $r^{\leftarrow}=$ $(U \times U) \backslash r^{-1}$.

Corollary 15. Let $r$ be a binary relation on a universe $U$. Then $r$ is serial (inverse serial) if and only if the corresponding direlation $(r, R)$ on the discrete texture $(U, \mathcal{P}(U))$ is serial (inverse serial).

Proof. Suppose that $r$ is a binary relation on a universe $U$ and $(r, R)$ is the corresponding direlation the discrete texture $(U, \mathcal{P}(U))$. It was shown [6] 
that $\left(r^{\leftarrow A} A, R^{\leftarrow A}\right)=\left(\underline{a p p}_{r} A, \overline{a p p}_{r} A\right)$ for all $A \in \mathcal{U}$. Then we have:

$$
\begin{aligned}
r \text { is serial } & \Longleftrightarrow \underline{a p p}_{r} A \subseteq \overline{a p p}_{r} A, \quad A \subseteq U \\
& \Longleftrightarrow r^{\leftarrow} A \subseteq R^{\leftarrow} A, \quad A \in \mathcal{P}(U) \\
& \Longleftrightarrow(r, R) \text { is serial. } \quad \square
\end{aligned}
$$

\section{Conclusion}

Rough sets are defined on the basis of lower and upper approximation operators obtained from the equivalence relations. In addition, generalized rough sets obtained from reflexive, transitive or serial relations are used in solving various problems in many areas as well as in topology.

Textural rough set algebra was introduced to approach for generalized rough set, and it is obtained effective results for classical rough sets. On the other hand, ditopologies (dichotomous topologies) on textures unify the fuzzy topologies, topologies and bitopologies in a non-complemented setting by means of duality in the textural concepts [5].

In this study, serial direlations are defined and their algebraic properties are investigated. In further studies, the conditions for obtaining a ditopology from the serial direlation and the algebraic properties of serial textural rough sets can be examined.

\section{REFERENCES}

[1] Brown, L. M. and Diker, M. Ditopological texture spaces and intuitionistic sets, Fuzzy sets and systems, 98 (1998), 217-224.

[2] Brown, L. M. and Ertürk, R. Fuzzy Sets as Texture Spaces, I. Representation Theorems, Fuzzy Sets and Systems, 110 (2) (2000), 227-236.

[3] Brown, L. M. and Ertürk, R. Fuzzy sets as texture spaces, II. Subtextures and quotient textures, Fuzzy Sets and Systems, 110 (2) (2000), 237-245.

[4] Brown, L. M., Ertürk, R. and Dost, Ş. Ditopological texture spaces and fuzzy topology, I. Basic Concepts, Fuzzy Sets and Systems, 147 (2) (2004), 171-199.

[5] Brown, L. M., Ertürk, R. and Dost, Ş. Ditopological texture spaces and fuzzy topology, II. Topological Considerations, Fuzzy Sets and Systems, 147 (2) (2004), 201-231.

[6] Diker M. Textural approach to generalized rough sets based on relations, Information Sciences, 180 (2010), 1418-1433.

[7] Diker M. Textures and Fuzzy rough sets, Fundamenta Informaticae, 108 (2011), 305336.

[8] Diker M. Definability and textures, International Journal of Approximate Reasoning, 53 (2012), 558-572.

[9] Diker M. Altay Uğur, A. Textures and covering based rough sets, Information Sciences, 184 (2012), 44-33. 
[10] Dost, Ş. Semi open and semi closed sets in ditopological texture space, Journal of Advanced Mathematical Studies, 5 (2012), 97-110.

[11] Dost, Ş. Precise Sets in Approximation Spaces and Textures, Fundamenta Informaticae, 158 (4) (2018), 353-368.

[12] Ekmekçi, R. Graded Diextremities, Filomat 32 (1) (2018), 149-164.

[13] Ekmekçi, R., Ertürk, R. Q-Convergence in graded ditopological texture spaces, Mathematica Moravica, 21 (1) (2017), 27-36.

[14] Pawlak, Z. Rough sets, International journal of computer and information sciences, 1982, 341-356.

[15] Qin, K., Gao, Y., Pei, Z. On covering rough sets, Lecture notes in computer science, 4481 (2007), 34-41.

[16] Qin, K., Yang, J., Pei,Z. Generalized rough sets based on reflexive and transitive relations, Information sciences, 178 (2008), 4138-4141.

[17] Yao, Y. Y. Two views of theory of rough sets in finite universes, International Journal Approximate reasoning, 15 (1996), 291-317.

[18] Yao, Y. Y. Constructive and algebraic methods of the theory of rough sets, Information Sciences, 109 (1998), 21-47.

[19] Zhu, W, Wang, F. Binary relation base rough set, Lecture notes in computer science, 4223 (2006), 276-285.

\author{
ŞENOL Dost \\ HaCetTepe University \\ Department of Secondary Science \\ and Mathematics Education \\ 06800 BEYTEPE \\ ANKARA \\ TURKEY \\ E-mail address: dost@hacettepe.edu.tr
}

